INTERNATIONAL JOURNAL OF MULTIDISCIPLINARY RESEARCH AND ANALYSis

ISSN(print): 2643-9840, ISSN(online): 2643-9875

Volume 04 Issue 11 November 2021

DOI: 10.47191/ijmra/v4-i11-36, Impact Factor: 6.072

Page No.- $1710-1712$

\title{
Arterial Hypotension Epidemiology in Adolescents and Youth in Fergana
}

\author{
T. S. Nurmatova ${ }^{1}$, N. S. Mamasoliyev ${ }^{2}$, Kh. Kh. Tursunov ${ }^{3}$, Z. M. Utanov ${ }^{4}$ \\ ${ }^{1} \mathrm{MD}$, Cardiologist at the Cardiology Department of the Namangan Branch of the Republican Scientific Center for Emergency \\ Medical Care \\ ${ }^{2}$ Doctor of Medical Sciences, Professor, Head of the Department of Internal Medicine, Cardiology and Emergency Medicine, Faculty \\ of Advanced Training and Retraining of Physicians, Andijan State Medical Institute, Andijan, Uzbekistan \\ ORCID: 0000-0002-5013 9647 \\ 3Doctor of Medical Sciences, Docent, Department of Internal Medicine, Cardiology and Emergency Medicine, Faculty of Advanced \\ Training and Retraining of Physicians, Andijan State Medical Institute, Andijan, Uzbekistan \\ ${ }^{4} \mathrm{MD}$, Assistant of the Department of Rehabilitation, Sports Medicine and Physical Culture, Faculty of Dentistry, Andijan State \\ Medical Institute, Andijan, Uzbekistan
}

ORCID: 0000-0002-9415-9308

ABSTRACT: The study analyzed data from a representative sample of adolescents and young men, 1465 people involved in a onestage epidemiological survey. The prevalence of arterial hypotension (AH) was 18.6\% (among adolescents - 10.8\% and among youth - 45.2\%; $\mathrm{P}<0.001$ ).

The revealed results should be considered when developing and implementing preventive programs for adolescent and young populations living in the regions of Uzbekistan.

KEYWORDS: Epidemiology, Prevalence, Arterial Hypotension (AH), Adolescents, Young Men

\section{INTRODUCTION}

It is well acknowledged that the significance of epidemiological researches in the realization of scientific and practical tasks for the prevention of cardiovascular diseases (CVDs) is becoming increasingly significant around the world. This is due to the fact that CVDs are the non-infectious illnesses that inflict the most harm to the modern population [1,3]. The adoption of epidemiological study results as the primary basis of CVD prevention might considerably lower "endpoints" in the community and/or in patients in this category $[2,4,5]$. In Uzbekistan, an increase in the prevalence of CVDs and mortality from them has been observed for several years. Obviously, the search for new population prophylactic approaches, which would reduce the "solid endpoints" in the population in CVDs, is an urgent scientific task.

The purpose of this study is to investigate the epidemiology of arterial hypotension among adolescents and youth in Uzbekistan

\section{MATERIALS AND METHODS}

Results of surveys of random, representative samples from teenagers (15-17 years old) and adolescents (18-22 years old) in the Fergana Valley of Uzbekistan served as the material for the current study.

A standardized one-stage epidemiological survey of teenage and adolescent populations (total number of those surveyed was 1465, response rate $97.8 \%$ ) in the Namangan region of the Fergana Valley was conducted according to a unified protocol. The research is a subset of an epidemiological study that aims to explore the epidemiological aspects of the primary chronic noninfectious illnesses in Uzbekistan's Fergana Valley. This article examines the findings in terms of the epidemiology of arterial hypotension $(\mathrm{AH})$.

Arterial hypotension was diagnosed when blood pressure $\leq 100 / 60 \mathrm{mmHg}$ according to WHO criteria (1989).

SAS, SPSS, and EXCEL-2000 statistical analysis programs were used to process and evaluate the data. 


\section{Arterial Hypotension Epidemiology in Adolescents and Youth in Fergana}

\section{RESULTS AND DISCUSSION}

A comparative analysis of AH prevalence rates showed that about $18.6 \%$ of adolescents and youth suffer from this disease. This corresponds to a high risk of cardiovascular complications. It turned out that every tenth adolescent $(10.8 \%)$ and $45.2 \%$ of young have AH ( $\mathrm{P}<0.001)$. These data assert that the situation in the regions of Uzbekistan in terms of the probability of developing cases of $\mathrm{AH}$ among adolescents and youth and the prevention of this pathology is undoubtedly urgent.

The frequency of primary and secondary AH in the $17-22$ year old group was $12.9 \%$ and $5.6 \%$, respectively (P0.01). The youth had a greater prevalence of primary and secondary $\mathrm{AH}(27.4 \%$ and $17.6 \%$, respectively, $\mathrm{P}<0.05)$ than adolescents $(8.6 \%$ and $2.2 \%, \mathrm{P}<0.01)$.

The study showed that the prevalence of physiological $\mathrm{AH}$ in the population sample was $4.2 \%$ among $0.4 \%$ of adolescents and $17.0 \%$ among youth $(P<0.0001)$. Pathological $\mathrm{AH}$ was diagnosed in $14.4 \%$ of the examinees $(10.4 \%$ in adolescents and $28.2 \%$ in youth, $\mathrm{P}<0.01)$. The greatest prevalence of chronic $\mathrm{AH}$ was $11.5 \%$, significantly higher in youth $(26.8 \%)$ than in adolescents (6.8\%); $\mathrm{P}<0.001$. The overall incidence of acute $\mathrm{AH}$ was no more than $7.2 \%$, with $18.2 \%$ of cases diagnosed in youth and a comparatively lower prevalence in the 14-17 year old surveyed group (4.0\%); $\mathrm{P}<0.05$.

The study found that among 17-22 year old natives of Uzbekistan's Fergana Valley, the prevalence of neurocardiac hypotension is substantially greater in youth (27.7\%) than in adolescents (9.9\%); P0.01. This type of AH occurred at a frequency of no more than $13.7 \%$ in the overall sample.

The findings indicate that the prevalence of postprandial hypotension was $9.8 \%$ in the total sample of examinees, $-4.2 \%$ in adolescents, and $-28.6 \%$ in youth (P0.001). However, orthostatic hypotension $(\mathrm{OH})$ had a lower prevalence of $4.2 \%(3.1 \%$ among 15-17 year olds and $8.1 \%$ among $18-22$ year olds, P0.01). AH with transient ischemic attacks had very identical epidemiological features, with $4.8 \%$ in the total sample and $3.7 \%$ in adolescents and youth $(P<0.05)$.

The incidence of hypotension caused by cardio arrhythmia was rather higher in youth (6.3\%) compared to adolescents (2.2\%); $P<0.001$. In the general sample, its prevalence was 3.1\%. A comparable significant contribution to the likelihood of juvenile hypotension, according to our data, was made by the medication factor. Thus, the prevalence of drug-induced hypotension in the population sample was between $1.2 \%$ and $1.0 \%$ among adolescents, and $2.1 \%$ among youth $(P<0.01)$.

Overall, a relatively high distribution of AH was detected among adolescents and youth (Fig. 1).

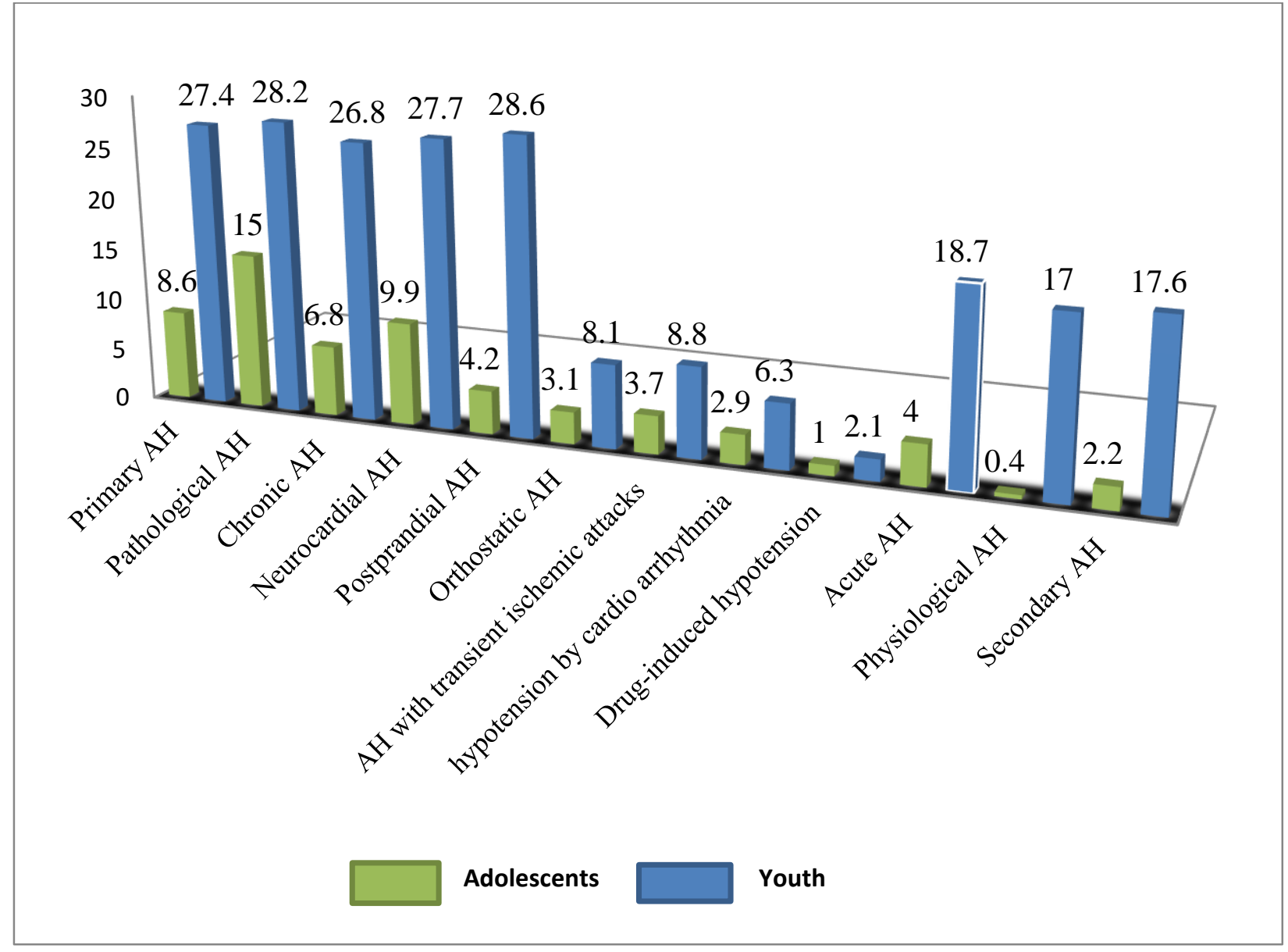

Figure 1. Distribution of arterial hypotension among studied adolescents and youth of Fergana Valley. 


\section{Arterial Hypotension Epidemiology in Adolescents and Youth in Fergana}

These findings should be considered in the development of regional AH prevention strategies for the adolescent and juvenile populations. The arterial hypotension prevention program should comprise a set of interventions that take into consideration special features of Uzbekistan's Fergana Valley areas and are directed at risk factors that negatively influence the population health of adolescents and youth. These preventive measures increase the targeting of prevention and are necessary to reduce the epidemiological endpoints from arterial hypotension in the general population of adolescents and young men in Uzbekistan.

\section{CONCLUSIONS}

1. A comparative analysis of the incidence of arterial hypotension among teenagers (10.8\%) and juveniles (45.2 \%) enabled us to identify geographical factors that should undoubtedly be considered when planning and executing comprehensive prevention programs.

2. It should be noted that comprehensive primary and secondary prevention of juvenile AH is not only the task of researchers and practical health care. Along with state budget allocations, the involvement of extra-budgetary sources of income of the region and local authorities to develop and implement a program of active prevention of $\mathrm{AH}$ and other chronic non-infectious diseases among adolescents and young adults is of particular importance.

\section{REFERENCE}

1) Drapkina O. M., Samorodskaya I.V. SCREENING: terminology, principles and international experience // VIDOX 2019. p. 9-14.

2) Boytsov S. A., Demkina A. E., Oshchepkova E. V., Dolgusheva Yu. A. Achievements and problems of practical karyology in Russia at the present stage // Cardiology. -2019; 59 (3). -p. 53-57.

3) Belyalov F. I. Cardiology // Practical Medicine. -Moscow. (2019) p.59-63.

4) Boytsov S. A., Pogosova N. B. et al. Cardiovascular prevention 2017. National guidelines. Russian Journal of Cardiology 2018; (6): p. 7-120.

5) Lopez A. D., Mathers C. D. et al. Global and regional burden of disease and risk factors, 2001: systematic analysis of population health data // Lancet 2006; 367: 1747-1753. 